\title{
ОБЕСПЕЧЕННОСТЬ ВИТАМИНОМ D У ПАЦИЕНТОВ С ПЕРВИЧНЫМ ГИПЕРПАРАТИРЕОЗОМ
}

\author{
Яневская Л.Г. ${ }^{,}$Погосян К.А. ${ }^{1}$, Каронова Т.Л. ${ }^{1,2}$ \\ 'Национальный медицинский исследовательский чентр им. В.А. Алмазова, Санкт-Петербург \\ Первый Санкт-Петербургский государственный медицинский университет им. акад. И.П. Павлова, \\ Санкт-Петербург
}

Определение уровня 25(OH)D у больных первичным гиперпаратиреозом (ПГПТ) является одним из важных диагностических моментов не только для исключения вторичного генеза, но и для профилактики послеоперационных осложнений. Целью исследования было изучить частоту определения 25(OH)D в сыворотке крови и уровень обеспеченности витамином D больных ПГПТ.

МАТЕРИАЛЫ И МЕТОДЫ: проанализированы истории болезни 449 пациентов с установленным диагнозом ПГПТ в период 2011-18 гг. Изучены данные анамнеза, лабораторных (ПТГ, 25(ОН)D, Са общ. и Са ион., фосфор, суточная кальциурия, ЩФ) методов исследования.

PЕЗУЛЬТАТЫ: из 449 больных с ПГПТ исходный уровень 25(ОН)D был определен только у 100 больных (22,3\%), которые были включены в дальнейший анализ данных. Медиана возраста больных была 61 год, медиана уровня 25(OH)D - 15,4 нг/мл, 93\% были женщины. Из 100 больных у 75\% имел место дефицит витамина D, у 15\% - недостаток и только 10\% имели нормальный уровень обеспеченности. Большинству пациентов 25(OH)D определялся непосредственно перед операцией, и только у троих нормализация показателей была достигнута на дооперационном этапе. Больные с нормальным уровнем 25(OH)D имели меньший ИМТ по сравнению с лицами с недостатком и дефицитом $(22,6$ \& 27,9 кг/м², p=0,013). Больные с дефицитом витамина D имели более высокий уровень ПтГ (231 \& 151 пг/мл соответственно, p=0,0001), Са ион. (1,52 \&1,43 ммоль/л, $\mathrm{p}=0,006)$ и Са общ. (2,82 \& 2,62 ммоль/л, $\mathrm{p}=0,003)$, чем лица с недостатком. Корреляционный анализ подтвердил наличие связи между 25(OH)D и ПТГ ( $r=-0,337, p=0,0007), C a$ общ. $(r=-0,296, p=0,009)$ и Са ион. ( $r=-0,218, p=0,001)$. Среди 100 больных четверо имели сочетание повышения уровня ПТГ с нормальным уровнем кальция. Показатели 25(OH)D свидетельствовали о наличии у них недостатка или дефицита витамина D, и ни у одного дефицит не был компенсирован.

ОБСУЖДЕНИЯ: несмотря на то, что определение уровня 25(OH)D входит в стандарт обследования больных ПГПТ, его уровень определялся только у каждого пятого больного, согласно полученным нами результатам. У подавляющего большинства больных имелся недостаток или дефицит витамина D на момент проведения хирургического лечения. Небольшая часть пациентов имела нормокальциемический вариант ПГПТ, для которых было характерно умеренное повышение ПТГ при нормальном уровне кальция крови. Однако даже у этих больных, не было проведено восполнение дефицита витамина D, а соответственно, не проведен дифференциальный диагноз первичного и вторичного генеза повышения ПтГ.

КЛЮЧЕВЫЕ СЛОВА: витамин D, гиперкальчиемия, ПТГ, кальчий крови, околощитовидные железы, первичный гиперnapamupeo3. 